\title{
A Hole Theory of Polymer Liquids and Glasses. III. Glass Transition and Glassy State; Heat Capacity Change at Glass Transition Temperature and Excess Enthalpy
}

\author{
Takuhei NosE \\ Department of Polymer Technology, Tokyo Institute of Technology, \\ Tokyo, Japan.
}

(Received June 25, 1970)

\begin{abstract}
In order to interpret the change in heat capacity $\Delta C_{p}$ at glass transition temperature and the excess enthalpy $H_{e}$ of polymer liquids and glasses relative to the crystals, a hole theory is presented in which both the hole fraction and the state of chain conformation are assumed to be frozen-in in the glassy state. In this treatment, $\Delta C_{p}$ and $H_{e}$ are both divided into two parts: one associated with intersegmental and the other with intrasegmental interactions. The relations $T V(\Delta \alpha)^{2} / \Delta C_{p}$ inter $\Delta \beta=1$ and $T V(\Delta \alpha)^{2} / \Delta C_{p} \Delta \beta \leq 1$ are derived, where $T$ is temperature, $V$ is volume, $\Delta \alpha$ and $\Delta \beta$ are the differences in the thermal expansion coefficient and compressibility between the liquid and glassy states respectively, and $\Delta C_{p}$ inter is the part of $\Delta C_{p}$ attributable to intersegmental interactions. Using the results of this theory, the experimental observations for $\Delta C_{p}$ and $H_{e}$ are compared and analyzed, bringing in at times the principle of corresponding states.
\end{abstract}

KEY WORDS Hole Theory/Polymer/Glass Transition/Heat Capacity / Excess Enthalpy /

In the preceding paper $^{1}$ we explained the glassy state by the hole theory, in which the glassy state was distinguished from the liquid and crystalline states by the freezing of holes which respect to intersegmental interactions. In this paper we deal with the thermodynamic properties which reflect both inter- and intra-segmental interactions, i.e., changes in heat capacity $\Delta C_{p}$ at glass transition temperature and "excess enthalpy" $H_{e}$ of the liquid and glass relative to the crystal, whereas in the previous paper $^{1}$ we considered the problems concerned with intersegmental interactions only, e.g., internal pressure. Since the glassy state may be regarded as a frozen liquid such that the polymer chains cannot change their conformations and that the segments cannot exchange their mean positions with each other, both holes and chain conformation are frozen-in in the glassy state. Thereforfore each of the above quantities $\Delta C_{p}$ and $H_{e}$ may consist of two elements: one associated with holes, intersegmental interactions, and the other associated with conformation of chain backbones, intrasegmental interactions.
Here we present a general hole theory incorporating the previous one, and derive a few relations between thermodynamic quantities such as $\Delta C_{p}, H_{e}, \Delta \beta$, and $\Delta \alpha$, where $\Delta \beta$ and $\Delta \alpha$ are respectively the differences in compressibility and and the thermal expansion coefficient between the liquid and glassy states. From the theoretical results we try to analyze the experimental observations, using at times the principle of corresponding states.

\section{THEORY}

\section{Model}

We consider a system in which $N$ segments are on $M$ lattice sites $(M \geq N)$. Thus there are $(M-N)$ empty sites or holes. If we denote the cell volume by $v^{*}$, then $M / N=V / N v^{*} \equiv V / V^{*} \equiv \tilde{V}$, where $V$ is volume, and hence the quantity $\left(1-\tilde{V}^{-1}\right)$ represents the hole fraction. In developping the theory further, we make the following two assumptions.

Assumption 1. The change in the cell volume with pressure and temperature is essentially independent of the hole fraction or $\tilde{V}$. This 


\section{T. Nose}

implies that the thermal expansion coefficient and compressibilility of the cell volume are almost continuous at the glass transition, and that the glasses of different hole fractions have almost the same cell volume, the same thermal expansion coefficient and the same compressibility (see APPENDIX in the preceding paper ${ }^{1}$ ).

Assumption 2. The free energy of the system is expressed by the sum of two kinds of free energy: one associated with the internal degrees of freedom or intrasegmental interactions, including the so-called short range interaction related to the chain conformation, and the other associated with the external degrees of freedom or intersegmental interactions. The former is considered to be independent of volume, and depends on temperature alone in the zero-th approximation. On the other hand, the latter should be dependent upon the hole fraction, the mean distance between nearest neighbor segments and temperature $T$. Hence it can be regarded as a function of $\tilde{V}, V^{*}$, and $T$. Thus the assumption is represented by

$$
\begin{aligned}
F= & U-T S=U_{1}(T)-T S_{1}(T) \\
& +U_{2}\left(\tilde{V}, V^{*}, T\right)-T S_{2}\left(\tilde{V}, V^{*}, T\right)
\end{aligned}
$$

where $U$ is the internal energy and $S$ the entropy, and the subscripts 1 and 2 refer to intrasegmental interactions, respectively. In general $U_{1}$ and $U_{2}$, or $S_{1}$ and $S_{2}$ cannot be expressed separately since, for example, the admissible number of arrangements of the polymer chain on the lattice and the conformational state of chain backbones may be mutually affected. However Gibbs and DiMarzio ${ }^{2}$ have shown, with a lattice theory, the possiblity that the partition functions for intra- and inter-molecular interactions are formally expressed separately. Thus we may reasonably use the expression of eq 1 in zero-th approximation. The free energy in our previous paper ${ }^{1,3}$ was also expressed in the form of eq 1 ; the partition function corresponding to $U_{1}-T S_{1}$ was represented by the implicit form $J(T)$, whereas that corresponding to $\left(U_{2}-T S_{2}\right)$ was given in the explicit form. The preceding model showed that Assumption 1 is valid at pressure much less than internal pressure and that the dependence of the $T-P-V^{*}$ relation on $\tilde{V}$ is only slight even at higher pressure.
In the glassy state, which is considered here to be a quasi-equilibrium state, it is assumed that the holes and the conformation of the chain backbones are completely frozen in. Thus, in the glassy state, the hole fraction or $\tilde{V}$ is assumed to be constant and the part of $U_{1}-T S_{1}$ associated with the hindered rotation about chain backbones is also assumed not to vary. On the other hand, in the liquid state, $\tilde{V}$ and $V^{*}$ are both allowed to change so as to minimize the free energy.

In the present theory in which inter- and intra-segmental interactions are separately expressed, both $\Delta C_{p}$ and $H_{e}$ are also represented by the sum of two parts:

$$
\begin{aligned}
\Delta C_{p} & =\Delta C_{p}^{\text {inter }}+\Delta C_{p}^{\text {intra }} \\
H_{e} & =H_{e}^{\text {inter }}+H_{e}^{\text {intra }}
\end{aligned}
$$

where superscripts inter and intra refer to interand intra-segmental interactions, respectively. From the definitions for these quantities, we have

$$
\begin{aligned}
\Delta C_{p} & =\Delta\left(\partial H_{e} / \partial T\right)_{p}, \\
\Delta C_{p}^{\text {inter }} & =\Delta\left(\partial H_{e}^{\text {inter }} / \partial T\right)_{p}, \\
\Delta C_{p}^{\text {intra }} & =\Delta\left(\partial H_{e}^{\text {intra }} / \partial T\right)_{p},
\end{aligned}
$$

where the symbol $\Delta$ denotes the difference between the liquid and glassy states. Since the the equation of state and $\Delta C_{p}$ inter are both determined by a volume-dependent part of free energy, some relation among $\Delta C_{p}$ inter,$\Delta \beta$ and $\Delta \alpha$ is expected, which will be derived in the next section. The expressions for $\Delta C_{p}$ inter and $H_{e}{ }^{\text {inter }}$ will be also derived by using the explicit form for $U_{2}$ given in the previous paper. ${ }^{1,3}$ Finally, we will present a simple rotational-isomeric model for the conformational energy of a chain backbone and express $\Delta C_{p}{ }^{\text {intra }}$ and $H_{e}{ }^{\text {intra }}$ with two parameters involved in the model.

$\Delta C_{p}$ inter and $H_{c}$ inter

First we will express the the heat capacity $C_{p}$ inter at constant pressure arising from intermolecular interactions. From the definition of the heat capacity, we have

$$
\begin{aligned}
C_{p 1}^{\text {inter }}= & T\left(\partial S_{2} / \partial \tilde{V}\right)_{V^{*}, T}(\partial \tilde{V} / \partial T)_{p}{ }^{1} \\
& +T\left(\partial S_{2} / \partial V^{*}\right)_{\tilde{V}, T}\left(\partial V^{*} / \partial T\right)_{p}{ }^{1}+T\left(\partial S_{2} / \partial T\right)_{\tilde{V}, V^{*}} \\
= & T\left(\partial S_{2} / \partial \tilde{V}\right)_{V^{*}, T} \tilde{V}\{\partial \ln V / \partial T)_{p}{ }^{1} \\
& \left.-\left(\partial \ln V^{*} / \partial T\right)_{p}{ }^{1}\right\}+T\left(\partial S_{2} / \partial V^{*}\right)_{\tilde{V}, T} \\
& \times\left(\partial \ln V^{*} / \partial T\right)_{p}{ }^{1}+T\left(\partial S_{2} / \partial T\right)_{\tilde{V}, V^{*}}
\end{aligned}
$$


A Hole Theory of Polymer Liquids and Glasses. III

for the liquid state, and

$C_{p g}^{\text {inter }}=T\left(\partial S_{2} / \partial V^{*}\right)_{\tilde{V}, T}\left(\partial V^{*} / \partial T\right)_{\tilde{V}, P}+T\left(\partial S_{2} / \partial T\right)_{\tilde{V}, V^{*}}$

for the glassy state, where the symbols 1 and $\mathrm{g}$ as subscripts and superscripts refer to the liquid and glassy states respectively. From eq 4 and 5 , we can derive $\Delta C_{p}^{\text {inter }}$ as

$\Delta C_{p}{ }^{\text {inter }} \equiv C_{p 1}^{\text {inter }}-C_{p \mathrm{~g}}^{\text {inter }}=T\left(\partial S_{2} / \partial \tilde{V}\right)_{V^{*}, T} \tilde{V}\left(\alpha_{1}-\alpha_{\mathrm{g}}\right)$

using the relation $\left(\partial \ln V^{*} / \partial T\right)_{p}{ }^{1}=\left(\partial \ln V^{*} / \partial T\right)_{\tilde{V}, P}$ $=\alpha_{\mathrm{g}}$ (Assumption 1), where $\alpha_{1}$ and $\alpha_{\mathrm{g}}$ are the thermal expansion coefficients $(\partial \ln V / \partial T)_{p}$ in the liquid and glassy states. The relation $P=$ $-(\partial F / \partial V)_{T}=-(\partial F / \partial V)_{V^{*}, T}=\left\{T\left(\partial S_{2} / \partial \tilde{V}\right)_{V^{*}, T}-\left(\partial U_{2} /\right.\right.$ $\left.\partial \tilde{V})_{V^{*}, T}\right\} / V^{*}$ can be derived from the condition that the volume of the cell is determined so as to minimize the free energy of the system (see APPENDIX of the preceding paper ${ }^{1}$ ). With this relation, $\Delta C_{p}$ inter may be expressed in an alternative form

$$
\Delta C_{p}^{\text {inter }}=\left\{\left(\partial U_{2} / \partial \tilde{V}_{V^{*}, T}\right)+P V^{*}\right\}\left(\alpha_{1}-\alpha_{\mathrm{g}}\right)
$$

Next we will express the quantity $\left(\partial S_{2} / \partial \tilde{V}\right)_{V, * T}$ in terms of $\alpha$ and compressibility $\beta \equiv-(\partial \ln V /$ $\partial P)_{T}$ in order to relate $\Delta C_{p}$ inter to the changes of $\alpha$ and $\beta$ at glass transition temperature. Thermal pressure coefficient $(\partial P / \partial T)_{V} \equiv \alpha / \beta$ is given by

$$
\begin{aligned}
(\partial P / \partial T)_{V}{ }^{1}= & \alpha_{1} / \beta_{1} \equiv(\partial S / \partial V)_{T}^{1} \\
= & \left(\partial S_{2} / \partial \tilde{V}\right)_{V^{*}, T} / V^{*}+\left\{\left(\partial S_{2} / \partial V^{*}\right)_{\tilde{V}, T}\right. \\
& -\left(\tilde{V} / V^{*}\right)\left(\left(\partial S_{2} / \partial \widetilde{V}\right)_{V^{*}, T}\right\}\left(\partial V^{*} / \partial V\right)_{T}{ }^{1}
\end{aligned}
$$

for the liquid state and

$$
\begin{aligned}
(\partial P / \partial T)_{V} \mathrm{~g} & =\alpha_{\mathrm{g}} / \beta_{\mathrm{g}}=(\partial S / \partial V)_{T}^{\mathrm{g}} \\
& =\left(\partial S_{2} / \partial V^{*}\right)_{\tilde{V}, T}\left(\partial V^{*} / \partial V\right)_{\tilde{V}, T} \\
& =\left(\partial S_{2} / \partial V^{*}\right) \tilde{V}, T / \tilde{V}
\end{aligned}
$$

for the glassy state.

Accordingly, combining eq 8 and 9 , and using the relation $\widetilde{V}\left(\partial V^{*} / \partial V\right)_{T}=\beta_{\mathrm{g}} / \beta_{1}$ (Assumption 1), we obtain

$$
\left(\partial S_{2} / \partial \tilde{V}\right)_{V^{*}, T} / V^{*}=\Delta \alpha / \Delta \beta
$$

where $\Delta \alpha=\alpha_{1}-\alpha_{\mathrm{g}}$ and $\Delta \beta=\beta_{1}-\beta_{\mathrm{g}}$.

Finally, substituting eq 10 into eq 6 , we have the expression

$$
\Delta C_{p}{ }^{\text {inter }}=T V(\Delta \alpha)^{2} / \Delta \beta
$$

If $\Delta C_{p}$ inter is replaced by $\Delta C_{p}$, this equation becomes identical with the well known equation $\Delta C_{p} \Delta \beta / T V(\Delta \alpha)^{2}=1$ which holds for a thermodynamical second order transition. In the present theory, since $\Delta C_{p}{ }^{\text {intra }}=\Delta C_{p}-\Delta C_{p}$ inter $^{2} \geq 0$, it follows that

$$
T V(\Delta \alpha)^{2} / \Delta C_{p} \Delta \beta=\Delta C_{p}{ }^{\text {inter }} / \Delta C_{p} \leq 1 .
$$

Heat capacity at constant pressure $C_{p}$ and that at constant volume $C_{v}$ are related to each other by the equation $C_{p}=C_{v}+T V \alpha^{2} / \beta$. Therefore we have

$$
\Delta C_{p}{ }^{\text {inter }}=\Delta C_{v}{ }^{\text {inter }}+\Delta\left(T V \alpha^{2} / \beta\right)
$$

noting $\Delta C_{p}^{\text {intra }}=\Delta C_{v}{ }^{\text {intra }}$ from Assumption 2 . From eq 11 and 13, we have

$$
\begin{aligned}
\frac{\Delta C_{v}{ }^{\text {inter }}}{\Delta C_{p}{ }^{\text {inter }}} & =\frac{\left(1-\alpha_{1} \beta_{\mathrm{g}} / \alpha_{\mathrm{g}} \beta_{1}\right)^{2}}{\left(\beta_{\mathrm{g}} / \beta_{1}\right)\left(\alpha_{1} / \alpha_{\mathrm{g}}-1\right)^{2}} \\
& =\frac{\left(1-\boldsymbol{P}_{\mathrm{i} 1} / \boldsymbol{P}_{\mathrm{ig}}\right)^{2} \boldsymbol{P}_{\mathrm{i} 1} \alpha_{\mathrm{g}}}{\left(\alpha_{1} / \alpha_{\mathrm{g}}-1\right)^{2} \boldsymbol{P}_{\mathrm{ig}} \alpha_{1}}
\end{aligned}
$$

where $P_{\mathrm{i}}$ is internal pressure defined by $(\partial U / \partial V)_{T}$ and the relation $P_{\mathrm{i}}=T \alpha / \beta-P=T \alpha / \beta$ is used. As can be seen from this equation, $\Delta C_{v}$ inter is not zero but positive, and thus it follows that $\Delta C_{v}>\Delta C_{v}$ intra.

According to the previous hole theory, ${ }^{1,3}$ the expression for $U_{2}$ is given by

$$
U_{2}=-N \varepsilon^{*} z^{\prime} K s(\tilde{V}-1+s)^{-1}
$$

where $z^{\prime}$ is the mean coordination number of a lattice site, i.e., $s=z^{\prime} \mid z$, and $-\varepsilon^{*} z K$ is the lattice energy, intersegmental interaction energy per segment, represented by

$-\varepsilon^{*} z^{\prime} K=\varepsilon^{*} z^{\prime}\left\{1.0109\left(\sigma^{3} N / V^{*}\right)^{4}-2.409\left(\sigma^{3} N / V^{*}\right)^{2}\right\} / 2$

Here the symbols $\varepsilon^{*}$ and $\sigma$ are the parameters involved in the Lennard-Jones 12-6 potential expressed as $u\left(r_{i j}\right)=4 \varepsilon^{*}\left\{\left(\sigma / r_{i j}\right)^{12}-\left(\sigma / r_{i j}\right)^{6}\right\}$ where $u\left(r_{i j}\right)$ is the potential energy of interaction between segments $i$ and $j$ as a function of separation $r_{i j}$. If we use the expression in eq 15 for $U_{2}$, the quantity $\Delta C_{p}{ }^{\text {inter }}$ is derived from eq 7 as

$$
\Delta C_{p}{ }^{\text {inter }}=N \varepsilon^{*} z^{\prime} K s\left(\tilde{V}_{\mathrm{g}}-1+s\right)^{-2} \tilde{V}_{\mathrm{g}} \Delta \alpha
$$

at atmospheric pressure $(P=0)$. 
We note here that, if the corresponding states principle for polymer liquids ${ }^{4}$ is assumed, $\Delta C_{p}$ inter of a molar unit having three external degrees of freedom, which is denoted here by $\Delta C_{p}$ 'inter may be a universal function of the reduced variables, as shown in the following. We let $3 c$ designate the external degrees of freedom per segment. Then, $\Delta C_{p}{ }^{\prime}$ inter is expressed as $\Delta C_{p}{ }^{\text {inter }} N_{\mathrm{A}} / c N$, where $N_{\mathrm{A}}$ is Avogadro's number. The principle of corresponding states for the equation of state is assumed here, and $U_{2}$ is also assumed be expressed as $U_{2}=N \varepsilon^{*} z^{\prime} \times f\left(\tilde{V}, \tilde{T}, V^{*} /\right.$ $\sigma^{3} N$ ), where the function $f$ is a universal one and the reduced temperature $\tilde{T}$ is defined as $c k T / \varepsilon^{*} z^{\prime}$. It then follows that

$$
\begin{aligned}
\Delta C_{p}{ }^{\prime \text { inter }} & =\Delta C_{p}^{\text {inter }} N_{\mathrm{A}} / c N=\Delta\left(\partial U_{2} / \partial T\right)_{p} N_{\mathrm{A}} / c N \\
& =\frac{\varepsilon^{*} z^{\prime} N_{\dot{A}}}{c} \Delta(\partial f / \partial T)_{p} \\
& =R\left\{(\partial f / \partial \tilde{T})_{\tilde{p}}-(\partial f / \partial \tilde{T})_{\tilde{V}}, \tilde{p}\right\}
\end{aligned}
$$

where $R$ is $k N_{A}$, the gass constant, and $\widetilde{P}$ is the reduced pressure. The right side of eq 18 is a function of the reduced variables. Accordingly, provided that the reduced variables at glass transition temperature $T_{\mathrm{g}}$ are independent of the substance, which holds roughly as shown in the preceding report, ${ }^{1}$ the change in heat capacity $\Delta C_{p}$ inter at $T_{\mathrm{g}}$ for a molar unit having three external degrees of freedom should have the same value for any substance. This result is identical with the statement presented by Wunderlich ${ }^{5}$ based on the hole theory of Hirai and Eyring. ${ }^{5}$ In Wunderlich's treatment, however, the choice of a unit seems uncertain, whereas in the present case such a structural unit may be determined, for example, by the method described in the previous paper. ${ }^{3}$ Transforming eq 11, we obtain the expression for $\Delta C_{p}{ }^{\text {'inter }}$ as

$$
\begin{aligned}
\Delta C_{p}{ }^{\prime \text { inter }}= & R \tilde{T}_{\mathrm{g}} \tilde{V}_{\mathrm{g}}{ }^{\prime}\left(\Delta \tilde{\alpha}^{\prime}\right)^{2} / \Delta \tilde{\beta}^{\prime} \\
= & R \tilde{V}_{\mathrm{g}}{ }^{\prime} \Delta \tilde{\alpha}^{\prime}\left(1-\tilde{\alpha}_{\mathrm{g}}{ }^{\prime} / \tilde{\alpha}_{1}{ }^{\prime}\right) \widetilde{P}_{\mathrm{i} 1}^{\prime} \\
& \times\left\{1-\left(\tilde{\alpha}_{\mathrm{g}}{ }^{\prime} / \tilde{\alpha}_{1}{ }^{\prime}\right)\left(\widetilde{P}_{\mathrm{i} 1}^{\prime} / \widetilde{P}_{\mathrm{ig}}^{\prime}\right)\right\}^{-1}
\end{aligned}
$$

where $\tilde{V}^{\prime}, \tilde{\alpha}^{\prime}, \tilde{\beta}^{\prime}$, and $\widetilde{P}_{\mathrm{i}}{ }^{\prime}$ are defined as $V / \sigma^{3} N$, $\left(\partial \ln \tilde{V}^{\prime} / \partial \widetilde{T}\right)_{p}, \beta \varepsilon^{*} z^{\prime} / \sigma^{3}$, and $P_{\mathrm{i}} \sigma^{3} / \varepsilon^{*} z^{\prime}$, respectively (therefore $\widetilde{P}_{\mathrm{i}}^{\prime}=\tilde{T} \tilde{\alpha}^{\prime} / \widetilde{\beta}^{\prime}$ at $P=0$ ). On the other hand, it follows from eq 17 that an alternative form for $\Delta C_{p}{ }^{\prime \text { inter }}$ is given by

$$
\Delta C_{p}{ }^{{ }^{\text {inter }}}=R K s\left(\tilde{V}_{\mathrm{g}}-1+s\right)^{-2} \tilde{V}_{\mathrm{g}} \Delta \tilde{\alpha}^{\prime}
$$

This section will end with a convenient estimate of the excess enthalpy $H_{e}{ }^{\text {inter }}$ based on the expression for $U_{2}$ given by eq 15 with eq 16 . It is assumed here that a perfect crystalline state, the reference state, may be described as the case of $\tilde{V}=1$ in the hole model, i.e., as the case in which the hole fraction is zero. The excess enthalpy $H_{e}{ }^{\text {inter }}$ relative to the crystal may then be given as

$$
\begin{aligned}
H_{e}{ }^{\text {inter }} & \cong U_{1}^{\text {inter }}-U_{\mathrm{c}^{\text {inter }}} \\
& =-\varepsilon^{*} N z^{\prime} K\left\{s(\tilde{V}-1+s)^{-1}-1\right\} \\
& =\varepsilon^{*} N z^{\prime} K(\tilde{V}-1) /(\tilde{V}-1+s)
\end{aligned}
$$

for the liquid state, where $U_{1}$ inter is $U_{2}$ in the liquid state and $U_{\mathrm{c}}$ is that in the crystalline state, and

$$
H_{e}^{\text {inter }} \cong \varepsilon^{*} N z^{\prime} K\left(\tilde{V}_{\mathrm{g}}-1\right) /\left(\tilde{V}_{\mathrm{g}}-1+s\right)
$$

for the glassy state.

$C_{p}$ intra and $H_{e}{ }^{\text {intra }}-A$ Simple Model for the Conformational Energy of Chain Backbones

In this section, we will make a simple analysis of the change in heat capacity at $T_{\mathrm{g}}$ and the excess enthalpy attributable to intrasegmental interactions. Both these quantities may be related to the hindered rotation about polymer chain backbones, since other degrees of freedom of intrasegmental interactions may show almost the same features in different states, i.e., in the liquid, glassy, and crystalline states, at the same temperature.

We now represent the partition function $J(T)^{\mathrm{R}}$ for the hindered rotations about chain backbones with the simplest rotational-isomeric approximation as follows

$$
J(T)^{\mathrm{R}}=\left\{\sum_{i=1}^{n} w_{i} \exp \left(-\varepsilon_{i} / k T\right)\right\}^{m}
$$

where each unit of the main chain has $w_{i}$ rotational isomers which have the energy $\varepsilon_{i}$, hence $\sum_{i=1}^{n} w_{i}$ is the total number of rotational isomers for each unit, and $m$ is the total number of rotational units in the system. Thus the enthalpy, $H(T)^{\mathrm{R}}$ associated with chain conformation may be derived from the partition function, eq 23 , as

$H(T)^{\mathrm{R}}=-k T^{2}\left(\partial \ln J^{\mathrm{R}} / \partial T\right)$ 


$$
=m \sum_{i=1}^{n}\left\{w_{i} \hat{\varepsilon}_{i} \exp \left(-\varepsilon_{i} / k T\right)\right\} / \sum_{?=1}^{n} w_{i} \exp \left(-\varepsilon_{i} / k T\right)
$$

The heat capacity $C_{p}{ }^{\mathrm{R}}$ associated with the rotation of chain backbones may then be expressed as

$$
C_{p}{ }^{\mathrm{R}}=\left(\partial H^{\mathrm{R}} / \partial T\right)
$$

and the excess enthalpy $H_{e}{ }^{\text {intra }}$ as

$$
H_{e}^{\text {intra }}= \begin{cases}H(T)^{\mathrm{R}}-H_{\mathrm{c}}^{\mathrm{R}} & \text { in the liquid state } \\ H\left(T_{\mathrm{g}}\right)^{\mathrm{R}}-H_{\mathrm{c}}^{\mathrm{R}} & \text { in the glassy state }\end{cases}
$$

Here, $H_{\mathrm{c}}{ }^{\mathrm{R}}$ denotes $H^{\mathrm{R}}$ in the crystalline state, and it is assumed that in the glassy state the rotation about chain backbones is frozen-in in the conformational state at which the glass has been formed. Accordingly, $C_{p}{ }^{\mathrm{R}}$ of the grassy state is zero. Hence the quantity $\Delta C_{p}$ intra may be equal to $C_{p}{ }^{\mathrm{R}}$ of the liquid state at $T_{\mathrm{g}}$.

Considering the case of two isomers of different energies $(n=2)$, and assuming that in the crystalline state every rotational unit is in the conformational state $1(i=1)$, we have from eq 24 , 25 , and 26

$$
H_{e}^{\text {intra }}=m \Delta \varepsilon w \exp (-\Delta \varepsilon / k T) /\{1+w \exp (\Delta \varepsilon / k T)\}
$$

for the liquid state, and

$$
\begin{aligned}
\Delta C_{p}{ }^{\text {intra }}= & m k(\Delta \varepsilon / k T)^{2} w \exp (-\Delta \varepsilon / k T) / \\
& \times\{1+w \exp (-\Delta \varepsilon / k T)\}^{2}
\end{aligned}
$$

where $\Delta \varepsilon=\varepsilon_{2}-\varepsilon_{1}\left(\varepsilon_{1} \leq \varepsilon_{2}\right)$, and $w=w_{2} / w_{1}$. From these equations we readily derive the relations at $T_{\mathrm{g}}$ represented by

$$
\begin{aligned}
\Delta \varepsilon / k T & =T \Delta C_{p}^{\text {intra }} / H_{e}^{\text {intra }}+H_{e}^{\text {intra }} / m k T \\
w & =\left(\Delta H^{\text {intra }}\right)^{2} \exp (\Delta \varepsilon / k T) /\left(m k T^{2} \Delta C_{p}^{\text {intra }}\right)
\end{aligned}
$$

\section{ANALYSIS OF EXPERIMENTAL OBSER- VATIONS AND DISCUSSION \\ $\Delta C_{p}$ inter and $H_{e}{ }^{\text {inter }}$}

In Table I, we show the values of $T V(\Delta \alpha)^{2}$ / $\Delta C_{p} \Delta \beta$ for polymers calculated from published data. The value of $T V(\Delta \alpha)^{2} / \Delta C_{p} \Delta \beta$, which is $\Delta C_{p}$ inter $/ \Delta C_{p}$ according to the present theory (eq 12 ), is less than unity and about 0.6 in most cases. This fact agrees with the theoretical prediction represented by eq 12 . The fact that this ratio is less than unity implies, in general, that two or more "ordering parameters" are necessary to specify a glassy state. ${ }^{7}$ The present theory has essentially two ordering parameters: one is the hole fraction representing intersegmental interactions and the other represents the state of chain conformation.

We now evaluate $\Delta C_{p}{ }^{\prime \text { inter }}$ and $\Delta C_{v}{ }^{\prime}$ inter with the average values of the reduced variables for some polymers which were obtained in the preceding paper. ${ }^{1}$ Required values for the calculation are as follows: $\tilde{V}_{\mathrm{g}}{ }^{\prime}=1.083, V_{\mathrm{g}}{ }^{*} / \sigma^{3} N=$ $0.997, \quad \tilde{\alpha}_{\mathrm{g}}{ }^{\prime}=2.27, \quad \tilde{\alpha}_{1}{ }^{\prime}=5.10, \quad \widetilde{P}_{\mathrm{i} 1}^{\prime}=0.616$, and $\widetilde{P}_{\mathrm{ig}}^{\prime}=0.386 . \quad\left(\widetilde{P}_{\mathrm{i} 1}^{\prime}\right.$ and $\widetilde{P}_{\mathrm{ig}}^{\prime}$ are the averages for poly(vinyl acetate) $)^{8}$ and poly(methyl methacrylate).$\left.^{9}\right)$ Considering $\Delta C_{v}{ }^{\text {inter }} / \Delta C_{p}{ }^{\text {inter }}=\Delta C_{v}{ }^{\text {inter }} /$ $\Delta C_{p}{ }^{\prime}{ }^{\text {inter }}, \quad \alpha_{1} / \alpha_{\mathrm{g}}=\widetilde{\alpha}_{1}{ }^{\prime} / \widetilde{\alpha}_{\mathrm{g}}{ }^{\prime}$, and $\boldsymbol{P}_{\mathrm{i} 1} / \boldsymbol{P}_{\mathrm{ig}}=\widetilde{\boldsymbol{P}}_{\mathrm{i} 1}^{\prime} / \widetilde{\boldsymbol{P}}_{\mathrm{ig}}^{\prime}$, and using eq 14 and 19 with the above values, we have

$$
\begin{aligned}
& \Delta C_{p}{ }^{\prime} \text { inter }=3.61 R=7.18 \mathrm{cal} / \mathrm{mol} \\
& \Delta C_{v}{ }^{\text {inter }} \Delta C_{p}{ }^{\prime}{ }^{\text {inter }}=0.163_{5} \\
& \Delta C_{v}{ }^{\text {inter }}=1.17_{3} \mathrm{cal} / \mathrm{mol} \\
& \Delta C_{p}{ }^{\prime \text { inter }}-\Delta C_{v}{ }^{\prime}{ }^{\text {inter }}=\Delta\left(T V \alpha^{2} / \beta\right)=6.01 \mathrm{cal} / \mathrm{mol}
\end{aligned}
$$

On the other hand, using eq $14^{\prime}$ and 20 with eq 16 , we have

$$
\begin{aligned}
& \Delta C_{p}{ }^{\prime}{ }^{\text {inter }}=2.12 R=4.22 \mathrm{cal} / \mathrm{mol} \\
& \Delta C_{v}{ }^{{ }^{\text {inter }}} / \Delta C_{p}{ }^{{ }^{\prime} \text { inter }}=0.069_{4},
\end{aligned}
$$

where we used $s=10 / 12$ and the theoretical value 0.707 for $\boldsymbol{P}_{\mathrm{ig} /} / \boldsymbol{P}_{\mathrm{i} 1}$ calculated from the expression for $U_{2}$ of eq 15 in the preceding paper. ${ }^{1}$ The value of $\Delta C_{p}{ }^{\text {inter }}$ obtained from eq 20 is much lower than that from eq 19, probably because the model for $U_{2}$ (eq 15) gives the lower value of $\left(\partial U_{2} / \partial \tilde{V}\right)_{V^{*}, T}$. This may be also responsible for the fact that the constant cell volume model in the previous paper ${ }^{3}$ gives a lower value of $\boldsymbol{P}_{\mathrm{i} 1}$. The latter value for $\Delta C_{v}{ }^{\prime \text { inter }} / \Delta C_{p}{ }^{\prime}$ inter is much lower than the former because it is sensitive to the ratio $\boldsymbol{P}_{\mathrm{i} 1} / \boldsymbol{P}_{\mathrm{ig}}$, to which the theory gives a value lower than the experimental one. ${ }^{1}$

According to the present analysis, a considerable amount of $\Delta C_{v}{ }^{\prime}$ inter is expected and we cannot regard $\Delta C_{v}$ as the change in heat capacity $\Delta C_{v}$ intra associated with intrasegmental interactions only. In other words, the quantity $\Delta C_{v}$ may include the change in heat capacity arising from the changeability of the mean posi- 
Table I. Various thermodynamic

\begin{tabular}{|c|c|c|c|c|c|c|}
\hline Polymer & $\begin{array}{l}T_{\mathrm{g}} \\
{ }^{\circ} \mathrm{K}\end{array}$ & $\begin{array}{l}V_{\mathrm{g}} \\
\mathrm{cc} / \mathrm{g}\end{array}$ & $\begin{array}{r}\alpha_{1} \times 10^{4} \\
{ }^{\circ} K^{-1}\end{array}$ & $\alpha_{\mathrm{g}} \times 10^{4}$ & $\begin{array}{l}\beta_{1} \times 10^{5} \\
\mathrm{~cm}^{2} / \mathrm{kg}\end{array}$ & $\begin{array}{l}\beta_{\mathrm{g}} \times 10^{5} \\
\mathrm{cal} / \mathrm{deg} \mathrm{g}\end{array}$ \\
\hline Polystyrene $^{a}$ & 362 & 0.97 & $5.45^{b}$ & $2.46^{b}$ & $4.72^{b}$ & $3.16^{b}$ \\
\hline Poly(methyl methacrylate) ${ }^{b}$ & 384 & 0.855 & 5.52 & 2.58 & 5.07 & 3.84 \\
\hline Poly(vinyl chloride) $\left\{\begin{array}{l}\text { d } \\
\theta\end{array}\right.$ & $\begin{array}{l}353 \\
348\end{array}$ & $\begin{array}{l}0.75 \\
0.725\end{array}$ & $\begin{array}{l}3.76 \\
5.70\end{array}$ & $\begin{array}{l}1.61 \\
1.99\end{array}$ & $\begin{array}{l}3.89 \\
4.23\end{array}$ & $\begin{array}{l}3.30 \\
2.42\end{array}$ \\
\hline Poly(vinyl acetate) $\quad\left\{\begin{array}{l}g \\
h\end{array}\right.$ & $\begin{array}{l}294 \\
298\end{array}$ & $\begin{array}{l}0.836 \\
0.855\end{array}$ & ${ }^{5.93} \Delta \alpha$ & 2.28 & ${ }_{\Delta \beta}^{3.84}$ & $56^{i}$ \\
\hline
\end{tabular}

${ }^{\mathrm{a}}$ ref $10, \mathrm{~b}$ ref $9, \mathrm{c}$ ref $11, \mathrm{~d}$ ref 9 and $12, \mathrm{e}$ ref $13, \mathrm{f}$ ref $5, \mathrm{~g}$ ref $8, \mathrm{~h}$ ref $14, \mathrm{i}$ ref 15.

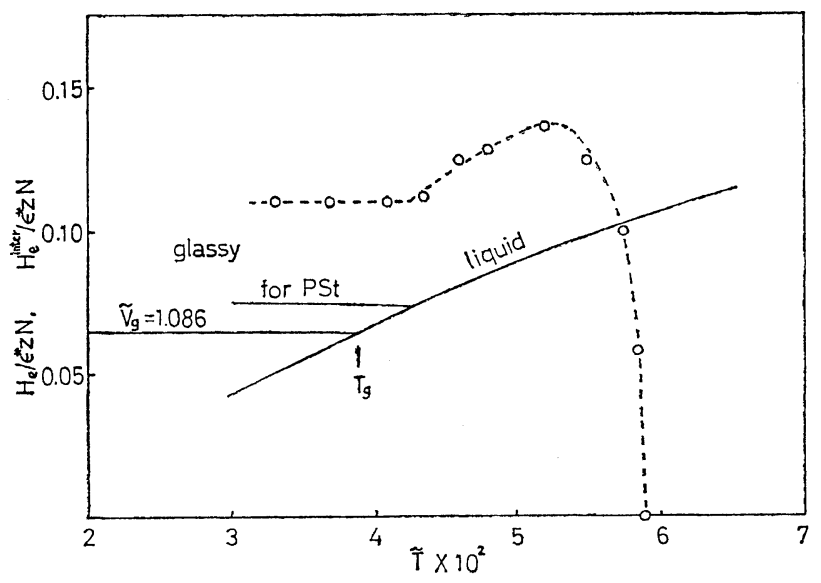

Figure 1. Calculated reduced excess enthalpy $H_{e}$ inter $/ \varepsilon^{*} z^{\prime} N$ associated with intersegmental interactions and experimental excess enthalpy $H_{e}$ of polysytyrene divided by $\varepsilon^{*} z^{\prime} N$ : curve for $H_{e} e^{\text {inter }} / \varepsilon^{*} z^{\prime} N$ calculated from eq 21 and $22 ; \bigcirc$, experimental value of $H_{e} / \varepsilon^{*} z^{\prime} N$ for polystyrene ${ }^{10}\left(\varepsilon^{*} z^{\prime} N=118 \mathrm{cal} / \mathrm{g}\right)^{3}$.

tion of the center of the segment in the liquid state, in addition to the change in heat capacity for intrasegmental degrees of freedom associated with the hindered rotation about main chains.

In Table I, the values of the change in various kinds of heat capacity at $T_{\mathrm{g}}$ for several polymers are shown, being calculated from published data with eq 11 and 14 . Such values as $\Delta C_{p}{ }^{\text {inter }}, \Delta C_{v}{ }^{\text {inter }}$, and $\Delta C_{p}{ }^{\text {intra }}$ are greatly dependent on the values of $\alpha_{\mathrm{g}} / \alpha_{1}$ and $\boldsymbol{P}_{\mathrm{ig}} / \boldsymbol{P}_{\mathrm{i} 1}$, and therefore it is very difficult to obtain these values accurately. For the same reason the constancy of the obtained values of $\Delta C_{p}{ }^{\prime}$ inter in Table $I$ is poor because the the principle of corresponding states for the equation of state is not sufficiently well satisfied.

The theoretical results for $H_{e}{ }^{\text {inter }}$ expressed by eq 21 and 22 are illustrated in Figure 1, being compared with the experimental total ex- cess-enthalpy for polystyrene ${ }^{10}$ derived by $\varepsilon^{*} N z^{\prime}$. The value of $\varepsilon^{*} N z^{\prime}$ used is that obtained in the previous paper, ${ }^{3}$ and the total excess enthalpy was estimated by converting the observed excess enthalpy relative to the $39-\%$ crystalline to $100-\%$. The calculated curve of $H_{e}^{\text {inter }} / \varepsilon^{*} z^{\prime} N$ for polystyrene glass is evaluated from eq 22 with $V_{\mathrm{g}} / V_{\mathrm{g}}{ }^{*}$ $=1.097$ given in the preceding report. ${ }^{1}$ The excess enthalpy $H_{e}$ inter arising from intersegmental interactions associated with the hole is about two thirds of the total excess enthalpy $H_{e}$. The remaining portion of the excess enthalpy $H_{e}-$ $H_{e}{ }^{\text {inter }}$ may be attributed to intrasegmental interactions, namely, the conformational energy of hindered rotation about chain backbones.

$\Delta C_{p}^{\text {intra }}$ and $H^{\text {intra }}$

Subtracting the calculated $H_{e}^{\text {inter }}$ from the observed $H_{e}$, we have the values of $H_{e}^{\text {intra }}$ for polystyrene and show them in Figure 2. The 
A Hole Theory of Polymer Liquids and Glasses. III

quantities at glass transition

\begin{tabular}{|c|c|c|c|c|c|}
\hline $\begin{array}{c}\Delta C_{p} \\
\mathrm{cal} / \mathrm{deg} \mathrm{g}\end{array}$ & $\begin{array}{l}\Delta C_{p}^{\text {inter }} \\
\text { cal/deg g }\end{array}$ & $\begin{array}{l}\Delta C_{p^{\prime}}^{\prime \text { inter }} \\
\text { cal } / \mathrm{mol}\end{array}$ & $\begin{array}{l}\Delta C_{v^{\text {inter }}} \\
\mathrm{cal} / \mathrm{deg} \mathrm{g}\end{array}$ & $\begin{array}{c}\Delta C_{p}^{\text {intra }}=\Delta C_{p}-\Delta C_{p}^{\text {inter }} \\
\text { cal } / \mathrm{deg} \mathrm{g}\end{array}$ & $\begin{array}{c}T V(\Delta \alpha)^{2} / \\
\Delta C_{p} \Delta \beta\end{array}$ \\
\hline 0.075 & $0.048_{6}$ & $7.0_{9}$ & 0.0050 & $0.026_{3}$ & 0.65 \\
\hline $0.086^{\mathrm{c}}$ & $0.056_{1}$ & $8.1_{4}$ & $0.011_{5}$ & $0.029_{9}$ & 0.65 \\
\hline $0.068^{\mathrm{f}}$ & $\begin{array}{l}0.033_{3} \\
0.048_{1}\end{array}$ & $=$ & $\begin{array}{l}0.0093 \\
0.0039\end{array}$ & $\begin{array}{l}0.034_{7} \\
0.019_{9}\end{array}$ & $\begin{array}{l}0.49 \\
0.71\end{array}$ \\
\hline $0.10^{\mathrm{h}}$ & $\begin{array}{l}0.052_{1} \\
0.063_{2}\end{array}$ & $\begin{array}{l}4.8_{4} \\
5.8_{7}\end{array}$ & 0.0040 & $\begin{array}{l}0.047_{0} \\
0.036_{8}\end{array}$ & $\begin{array}{l}0.52 \\
0.63\end{array}$ \\
\hline
\end{tabular}

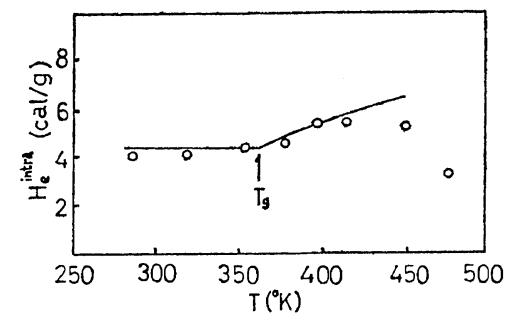

Figure 2. Excess enthalpy $H_{e}$ intra associated with intrasegmental interactions for polystyrene: curve of $H_{e}$ intra calculated from eq 27 with $\Delta \varepsilon=$ $1.76 \mathrm{kcal} / \mathrm{mol}$ and $w=1.76 ; \bigcirc$, values of $H_{e}$ inter calculated by substracting the calculated $H_{e}$ inter (eq 21 and 22) from the experimental $H_{e}{ }^{10}$

value of $H_{e}^{\text {intra }}$ at $T_{\mathrm{g}}, 362^{\circ} \mathrm{K}$, thus obtained is $4.49 \mathrm{cal} / \mathrm{g}$. The value of $\Delta C_{p}$ intra for polystyrene is estimated to be $0.026_{3} \mathrm{cal} / \mathrm{deg} \mathrm{g}$ as shown in Table I. Using eq 29 and 30 with these values of $H_{e}{ }^{\text {intra }}$ at $T_{\mathrm{g}}$ and $\Delta C_{p}{ }^{\text {intra }}$, and taking a half of a repeating unit as a rotational unit, we have $1.76 \mathrm{kcal} / \mathrm{mol}$ for $\Delta \varepsilon$ and 1.76 for $w$. With these values, $H_{e}^{\text {intra }}$ in the liquid state is calculated from eq 27 and illustrated by a solid line in Figure 2. The calculated curve shows the reasonable increase in $H_{e}^{\text {intra }}$ above $T_{\mathrm{g}}$. The value of $\Delta \varepsilon$ obtained here is fairly close to the $1.67 \mathrm{kcal} / \mathrm{mol}$ evaluated by Gibbs and DiMarzio ${ }^{2}$ with their theory of glass transition in which $w=2$. Although the physical meanings of $\Delta \varepsilon$ and $w$ obtained experimentally are not sufficiently clear due to the simplicity of the model, the values for $\Delta \varepsilon$ and $w$ obtained above appear to show reasonable magnitudes. Therefore the value of $H_{e}{ }_{e}^{\text {intra }}$ estimated as $H_{e}-H_{e}{ }^{\text {inter }}$ may reasonably be expected, and hence $H_{e}$ inter evaluated by eq 21 and 22 may also be said to give reasonable magnitude and temperature dependence.
Acknowledgment. The author would like to thank Prof. T. Hata of the Tokyo Institute of Technology for encouraging discussions and much useful advice.

\section{LIST OF SYMBOLS}

$C_{p}$, heat capacity at constant pressure

$C_{v}$, heat capacity at constant volume

$C_{p}{ }^{\prime}$, heat capacity at constant pressure of a molar unit having three external degrees of freedom

$C_{v}{ }^{\prime}$, heat capacity at constant volume of a molar unit having three external degrees of freedom

$c$, one third of external degrees of freedom

c, as subscript designate the crystalline state

$F$, Helmohltz free energy

$\mathrm{g}$, subscript and superscript for glassy state (except in $T_{\mathrm{g}}, \widetilde{T}_{\mathrm{g}}, V_{\mathrm{g}}, \widetilde{V}_{\mathrm{g}}{ }^{*}$, and $\widetilde{V}_{\mathrm{g}}{ }^{\prime}$, where $\mathrm{g}$ refers to glass transition point.)

$\mathrm{g}$, as subscript in $T_{\mathrm{g}}, \tilde{T}_{\mathrm{g}}, V_{\mathrm{g}}, \tilde{V}_{\mathrm{g}}, V_{\mathrm{g}}{ }^{*}$, and $\tilde{V}_{\mathrm{g}}^{\prime}$ designate the glass transition point.

$H_{e}$, excess enthalpy relative to the crystal

$H(T)^{\mathrm{R}}$, enthalpy associated with the hindered rotation about chain backbones

inter, superscript for intersegmental interactions intra, superscript for intrasegmental interactions $J(T)^{\mathrm{R}}$, partition function associated with the hindered rotation about chain backbones

$K$, function defined by eq 16

$k$, Boltzmann's constant

1, subscript and superscript for liquid state $M$, total number of lattice sites in system

$m$, total number of rotational units in system $N$, tatal number of segments in system

$N_{\mathrm{A}}$, Avogadro's number

$n$, number of rotational isomers having different energies per rotational unit 
$P$, pressure

$\widetilde{P}$, reduced pressure, $P \sigma^{3} / \varepsilon^{*} z^{\prime}$

$\boldsymbol{P}_{\mathrm{i}}$, internal pressure, $(\partial U / \partial V)_{T}$

$\widetilde{P}_{\mathrm{i}}{ }^{\prime}$, reduced internal pressure defined as $\widetilde{P}_{\mathrm{i}}{ }^{\prime}$ $\equiv P_{\mathrm{i}} \sigma^{3} / \varepsilon^{*} z^{\prime}$

$R$, gas constant per mole

$S$, entropy

$s$, ratio of cordination number of a segment to a lattice site, $z^{\prime} / z$

$T$, temperature

$\tilde{T}$, reduced temperature defined as $\tilde{T} \equiv$ $\operatorname{ckT} / \varepsilon^{*} z^{\prime}$

$U$, internal energy

$V$, volume

$\tilde{V}$, reduced volume defined as $\tilde{V}=V / V^{*}=$ $M / N$

$V^{*}$, volume of cells occupied by segments, $v^{*} N$

$\tilde{V}^{\prime}$, reduced volume defined as $\tilde{V}^{\prime}=V / \sigma^{3} N$

$v^{*}$, volume of a cell

$w$, ratio of $w_{2}$ to $w_{1}, w_{2} / w_{1}$

$w_{i}$, number of rotational isomers per rotational unit which have energy $\varepsilon_{i}$

$z$, coordination number of a lattice site

$z^{\prime}$; coordination number of a segment

$\alpha$, thermal expansion coefficient

$\tilde{\alpha}^{\prime}$, reduced quantity of thermal expansion coefficient defined as $\tilde{\alpha}^{\prime}=\left(\partial \ln \tilde{V}^{\prime} / \partial \tilde{T}\right)_{p}=$ $\alpha \varepsilon^{*} z^{\prime} / c k$

$\beta$, isothermal compressibility

$\tilde{\beta}^{\prime}$, reduced isothermal compressibility defined as $\tilde{\beta}^{\prime}=\beta \varepsilon^{*} z^{\prime} / \sigma^{3}$

$\Delta$, difference between the liquid and glassy states (except in $\Delta \varepsilon$ )

$\varepsilon^{*}$, potential energy at minimum of potential energy curve of Lennard-Jones 12-6 potential

$\varepsilon_{i}$, potential energy of rotational isomer $i$

$\Delta \varepsilon$, difference in potential energy between two rotational isomers 2 and $1, \varepsilon_{2}-\varepsilon_{1}$ $\left(\varepsilon_{2} \geq \varepsilon_{1}\right)$

$\sigma$, distance between segment centers at which potential energy is zero in Lennard-Jones $12-6$ potential

1 , as subscript designates the intrasegmental interactions (except in $\varepsilon_{1}$ and $w_{1}$ )

2 , as subscript designates the intersegmental interactions (except in $\varepsilon_{2}$ and $w_{2}$ )

\section{REFERENCES}

1. T. Nose, Polymer J., 2, 427 (1971).

2. J. H. Gibbs and E. A. DiMarzio, J. Chem. Phys., 28, 373, 807 (1958).

3. T. Nose, Polymer J., 2, 124 (1971).

4. I. Prigogine, "The Molecular Theory of Solutions", Interscience Publishers. Inc., New York, N.Y., 1957; P. J. Flory, R. A. Orwoll, and A. Vrij, J. Amer. Chem. Soc., 86, 3507 (1964).

5. B. Wunderlish, J. Phys. Chem., 64, 1052 (1960).

6. N. Hirai and H. Eyring, J. Appl. Phys., 29, 810 (1958); J. Polym. Sci., 73, 51 (1959).

7. R. O. Davies and G. O. Jones, Proc. Roy. Soc., Ser. A, 217, 26 (1953); Advan. in Phys., 2, 370 (1959).

H. Breuer and G. Rehage, Kolloid-Z, 216-217, 159 (1967).

8. G. Allen, G. Gee, D. Mangaraj, D. Sims, and G. J. Wilson, Polymer, 1, 467 (1960).

9. K. H. Hellwege, W. Knappe, and P. Lehmann, Kolloid-Z, 183, 110 (1962).

10. F. E. Karasz, H. E. Bair, and J. M. O'Reilly, J. Phys. Chem., 69, 2657 (1965).

11. J. M. O'Reilly, J. Polym. Sci., Part C, 14, 49 (1964).

12. G. Gee, Polymer, 7, 177 (1966).

13. P. Heydemann and H. D. Guicking, Kolloid-Z, 193, 16 (1963).

14. J. M. O'Reilly, J. Polym. Sci., 57, 445 (1962).

15. J. E. McKinney and H. V. Belcher, J. Res. Nat. Bur. Stand., 67A, 43 (1963). 